\title{
La distinción entre decir y mostrar y la superación de los problemas filosóficos
}

Kurt Wischin Universidad Nacional Autónoma de México

Resumen

Las dificultades que ofrece el Tractatus para la interpretación detallada de muchos de sus pasajes considerados clave, junto con la crítica que su autor ha formulado en su desarrollo filosófico posterior contra algunos aspectos de la visión del lenguaje de su primera obra, han tenido frecuentemente como consecuencia que los intérpretes pierdan de vista el objetivo general de la obra, y en qué grado la filosofía wittgensteiniana es movida por una misma preocupación filosófica durante toda la vida del pensador austrobritánico. Una discusión de las causas de la distinción clave del Tractatus entre lo que dicen y lo que muestran las oraciones sugiere que la visión que motiva esta distinción tractariana sigue vigente en la filosofía posterior de Wittgenstein.

Palabras clave: proposición, mostrar, decir, continuidad filosófica, sinsentido, límite.

\section{Abstract}

The difficulties encountered for a detailed interpretation of many a key passage of the Tractatus, together with the critical comments the author himself has leveled during his later philosophical development at some aspects of the vision of language in his first opus frequently has had the consequence that some interpreters lose sight of the general target of the work and the degree to which Wittgensteinian philosophy 
is moved by one and the same philosophical worries during the whole life of the austro-british thinker. An argument of the motives behind the key distinction of the Tractatus between what a proposition says and what it shows suggests that the vision that is the source of this tractarian distinction is forcefully at work also in the later philosophy of Wittgenstein.

Keywords: proposition, show, say, philosophical continuity, nonsense, limit.

$\mathrm{L}$ a meta del filosofar de Wittgenstein en su obra de juventud, así como en su pensar maduro, es la superación de los problemas filosóficos. No está enamorado de ellos. Los considera pseudoproblemas. Son problemas que no tienen solución, porque no son problemas reales. El filósofo debe hacernos ver que los enigmas filosóficos se deben a confusiones que nacen de nuestra práctica lingüística, y disolverlos. Pero no por ello son triviales, sino tan profundos como las raíces de nuestro lenguaje. No tener en cuenta este motivo desvía fácilmente la discusión e induce errores de apreciación de los diferentes aspectos de la obra.

El presente trabajo se propone ilustrar, con la ayuda del ejemplo de la distinción entre lo que dicen y lo que muestran las proposiciones - distinción omnipresente en el Tractatus LogicoPhilosophicus-, que diferentes maneras de dilucidación de estos aspectos pueden ayudar a ver este motivo unificador como el motor que impulsa la reflexión wittgensteiniana, u ofuscar la comprensión y convertir la discusión de estos aspectos en meras cuestiones técnicas, perdiendo así de vista el papel que juegan en la filosofía de Wittgenstein.

La parte introductoria de este trabajo da algunos ejemplos de la recepción de la filosofía de Wittgenstein, en particular del único libro que publicó en vida, el Tractatus. La segunda parte es un resumen de la explicación de la distinción entre decir y mostrar 
de las proposiciones, tal como la ofrece el erudito Hans-Johann Glock. La tercera y última parte da un ejemplo de una lectura más amplia de esta distinción para ilustrar cómo esta lectura nos permite conservar el panorama general del objetivo filosófico de Wittgenstein. La conclusión es que Glock está en lo cierto (al menos al nivel de discusión poco detallada que aquí podemos presentar), pero que es insuficiente si queremos comprender lo que motiva a Wittgenstein a hacer esta distinción más allá del propio sistema de lógica del que depende también.

\section{La importancia del Tractatus, y cómo no leerlo}

Michael Dummett, en su obra Origins of Analytical Philosophy, rastrea para nosotros las fuentes de la filosofía analítica desde la filosofía de habla alemana de finales del siglo XIX. Dummett identifica la filosofía analítica con la creencia "en primer lugar, de que una explicación filosófica del pensamiento puede obtenerse sólo mediante una explicación del lenguaje, y en segundo lugar, que una explicación de amplitud sólo puede lograrse así" (Dummett, 1996: 4). La mayor parte de esta obra consiste en una comparación de las teorías semánticas propuestas por Frege y Husserl. Para permitirnos visualizar lo sorprendente de sus observaciones acerca de la gran semejanza que exhiben estas propuestas —en vista de la enorme diferencia entre las filosofías analítica y fenomenológica que tomaron su respectivo desarrollo de estos principios-, Dummett invoca la imagen de dos grandes ríos: el Rin y el Danubio, los cuales tienen sus fuentes en regiones cercanas en el centro de Europa, pero desembocan en mares muy distantes entre sí. Pero luego dice algo que quizás nos podría sorprender aún más:

${ }^{1}$ Todas las traducciones al español son del autor del presente artículo. 
Si identificamos el giro lingüístico como el punto de arranque de la filosofía analítica propiamente dicha, entonces no puede haber duda de que, sea cual sea el grado en que Frege, Moore y Russell hayan preparado el terreno, el paso crucial fue tomado por Wittgenstein en el Tractatus Logico-philosophicus de 1922 (Dummett, 1996: 127).

Esto es notable porque muchos de los filósofos que suscriben ideas cuyo origen puede, al menos vagamente, atribuirse a la filosofía analítica, consideran el Tractatus un libro prácticamente inservible como fundamento de argumento filosófico alguno. Esta actitud es observable incluso en autores que, en general, parecen tener interés en promover el conocimiento y la comprensión de la filosofía wittgensteiniana. Robert Fogelin, por ejemplo, en su libro Wittgenstein observa que las ideas del alumno (y alguna vez amigo) de Russell sobre el fundamento lógico de la matemática carecen de suficiente elaboración para ser inteligibles:

Así, Wittgenstein invoca algún tipo de ideal constructivista y descarta las obras clásicas de la fundamentación de la matemática porque no cumplen con él. ¿Pero qué clase de constructivismo es? ¿Cómo se puede usar la definición de los números enteros para construir partes más amplias de la matemática...? Y otras preguntas más. Mientras no tengamos respuestas a preguntas de esta naturaleza, no tenemos idea en qué consiste la posición de Wittgenstein (Fogelin, 1987: 84).

La idea de que las propuestas lógicas del Tractatus sean ininteligibles por carecer de elaboración prevalecen aún hoy en día en la filosofía analítica. Gracias a esta vaguedad y falta de elaboración fue posible, parece suponer Fogelin, que por medio siglo pasara inadvertido el hecho de que, según él, ni siquiera es posible expresar mediante el lenguaje lógico propuesto por el Tractatus toda la 
lógica de primer orden, y dedica en la segunda edición del libro un capítulo entero al intento de demostrarlo. Ha habido varios esfuerzos por refutar las afirmaciones de Fogelin en este sentido, pero no fue sino hasta 1994 que el filósofo alemán Varga von Kibed demostró que la lectura que Fogelin hace de la propuesta lógica elaborada en el Tractatus se debe a una comprensión insuficiente de los objetivos de Wittgenstein.

El origen del problema de Fogelin es haber tenido una expectativa muy estrecha de lo que el Tractatus debiera ofrecer, a saber, un sistema de lógica formal explícito. Si éste hubiese sido el objetivo de Wittgenstein, el Tractatus, efectivamente, no ofrece sistema formal elaborado alguno; pero, en este sentido, el objetivo del Tractatus no es establecer un lenguaje formal, sino dejar en claro las condiciones necesarias de todo sistema lógico posible. Varga apunta:

Wittgenstein representa aquí... la forma general de la función veritativa como tal, que tiene que formar la base de todo sistema lógico posible. En cierto sentido, en esta forma general de la función veritativa, se encuentra condensada la forma del mundo, tal como Wittgenstein la ha desarrollado en las proposiciones previas del Tractatus (2001: 209).

El resto del artículo de Varga es una explicación detallada de por qué la crítica de Fogelin falla, y explica, además, por qué también la bien intencionada defensa del Tractatus de Scott Soames falla, y por qué la de Peter Geach, aunque presenta un punto de vista similar al del propio Varga, tampoco logra del todo su objetivo.

El problema de tratar adecuadamente el argumento del Tractatus, ejemplificado por la crítica de Fogelin, es típico de cierta actitud de analistas que, según creo, amenaza la comprensión de la filosofía de Wittgenstein. Para mencionar de paso otro ejemplo: en su crítica de la obra The Philosophy of Wittgenstein de G. Pitcher, 
Rush Rhees, por ejemplo, hace la siguiente observación acerca de lo que le parece una incomprensión completa de los motivos filosóficos que inspiraron la redacción del Tractatus:

No pienso que Pitcher jamás vio qué clase de cuestionamiento indujo a Wittgenstein a escribir el Tractatus; ni qué era aquello que Wittgenstein tenía la esperanza de que otros pudieran aprender de éste. Por ello, los comentarios finales significan poco para él. ¿Qué clase de conclusiones sugieren? ¿Qué dificultades de alcance profundo pudieran contrarrestarse de esta manera?

Las Investigaciones pudieran haberle ayudado. Pero él ve que Wittgenstein demuele su sistema y erige uno nuevo... Mientras que Wittgenstein hubiera demolido, de haber podido, la idea de discusión filosófica como competencia para determinar quién tiene razón y quién se equivoca (Rhees, 1996: 42).

Una noción importante para explorar los cuestionamientos filosóficos de Wittgenstein a que alude Rhees es la distinción entre lo que las proposiciones dicen y lo que muestran, distinción que es de primordial importancia para comprender el sistema del Tractatus. A pesar de la importancia de esta noción, no hay unanimidad entre los estudiosos de Wittgenstein de cómo dar cuenta de ella. En lo que sigue resumiré dos maneras de explicar la distinción entre decir y mostrar. La primera de ellas ve esta distinción como consecuencia de la teoría de lenguaje y del sistema lógico propuesto en el Tractatus. La segunda propone una lectura más amplia, $y$ ve en el problema que Wittgenstein trata de resolver, apelando a esta distinción, una clave para comprender toda la filosofía wittgensteiniana, no restringida al Tractatus; consecuentemente, se trata de un aspecto que revela la unidad del pensamiento de Wittgenstein, en lugar de tratarla como un aspecto que separe su filosofar de la juventud y de la madurez. 


\section{La distinción de decir y mostrar según Glock}

Hans-Johann Glock empieza su explicación de la relación decir I mostrar así: "La distinción entre lo que puede decirse mediante proposiciones significativas y lo que sólo puede ser mostrado, compenetra el Tractatus desde el prólogo a la advertencia final famosa 'de lo que no se puede hablar debe guardarse silencio"' (1996: 330). De acuerdo con Glock:

El Tractatus tiene, de hecho, dos partes, una lógica (ontología atomista, teoría pictórica, tautologías, matemática, ciencia) y una mística (solipsismo, ética, estética). El significado real de la distinción decir/mostrar se ubica en el hecho de que mantiene unidas las dos al proscribir tanto proposiciones sobre la esencia de la representación simbólica y aseveraciones místicas sobre el reino de valor (Glock, 1996: 330).

Una característica importante que comparte todo lo inefable tractariano es que no puede ser representado mediante proposiciones bipolares (verdaderas / falsas); es decir, mediante proposiciones que representan hechos posibles, como lo hacen las proposiciones científicas. Lo que generalmente se designa como la "teoría pictórica de lenguaje", desarrollada en el Tractatus, sostiene que las proposiciones representan hechos posibles en el mundo, porque proposición $\mathrm{y}$ hecho tienen la misma estructura lógica; y las proposiciones son verdaderas si el hecho se da, y falsas si no se da. Esto último es el significado del término "bipolar". Una proposición tiene que tener esta característica, de lo contrario no sería una proposición, sino, por ejemplo, una pseudo-proposición.

Los intentos por decir aquello que sólo puede ser mostrado tratan de decir lo que no pudiera ser de otra manera (por lo que no son expresables en proposiciones bipolares), y son, entonces, pseudo-proposiciones. Las "Pseudo-proposiciones no dependen de 
cómo son las cosas", dice Glock, "porque se relacionan con precondiciones 'trascendentales' de la representación y del mundo" (1996: 331). Lo místico a que Glock se refiere en el pasaje previo ocupa un lugar especial entre lo inefable, según Glock, porque ni siquiera existen proposiciones significativas que muestren, por ejemplo, valor ético. "A diferencia de lo lógico, lo místico es trascendente, no sólo trascendental" (Glock, 1996: 331). Glock no explica esta diferencia más allá del pasaje citado; este tipo de inefabilidad no se ajusta a la explicación que Glock da de otros aspectos de la distinción entre decir y mostrar, como él mismo aquí muy claramente expresa, ni explica en qué sentido esta distinción une las dos partes del Tractatus a que Glock alude en el pasaje citado. Espero hacer más plausible en la tercera sección del presente trabajo, porque pienso que esto es un defecto de la explicación de Glock.

Lo que hay de inefable, dejando a un lado lo anterior, tiene que ver con intentos por rebasar el límite de lo expresable, constituido por el propio sistema de representación. Por este motivo no es posible expresar en proposición significativa alguna ni la estructura del pensamiento ni la del mundo; ambas son condiciones de la posibilidad de representación:

[...] ninguna proposición puede decir algo sobre las propiedades lógicas del lenguaje: o bien, semejante proposición está conforme a la lógica, entonces estas proposiciones lógicas ya son entendidas (circularidad), o no [está conforme a la lógica], entonces no puede ser una proposición significativa (un lenguaje ilógico es imposible) (Glock, 1996: 332).

Es decir, representar los hechos y comprenderlos es uno, y se da en la lógica. Para poder explicar la lógica más allá de esto sería necesario suspenderla — cosa que es imposible- Este principio general revela también el motivo por el cual Wittgenstein insiste en que la forma que las proposiciones y lo que retratan comparten - la 
armonía entre pensamiento y realidad - no puede ser expresada en una proposición ( Wittgenstein, 1973: 2.172-2.174):

[ésta] no puede representarla como una posibilidad. Porque la forma pictórica de una proposición es una de sus 'propiedades internas' — no pudiera carecer de esta forma pictórica sin dejar de ser el retrato que es. Por lo mismo, ninguna otra proposición pudiera representarla como posibilidad, es decir, no puede haber proposiciones bipolares acerca de la forma pictórica de proposiciones (Glock, 1996: 332).

El principio de lo inefable de la forma lógica conlleva también la imposibilidad lógica de una teoría semántica, es decir, de expresar las relaciones lógicas entre las proposiciones, y tiene como consecuencia que los conceptos formales de la lógica son realmente pseudo-conceptos. Estas consideraciones también hacen superflua la teoría de tipos de Russell:

Según Wittgenstein semejante teoría ni es posible, ni necesaria. (1) ['La clase de leones es un león'] o bien es sobre signos — en este caso afirma un hecho contingente acerca de convenciones arbitrarias, no una regla lógica-; o trata de símbolos. En este caso debe referirse al sentido o significado de expresiones. Pero no puede referirse al sentido de (1), ya que esto, ex hypothesi, carece de sentido. Ni tampoco puede referirse al significado de los nombres que últimamente constituyen (1). Porque estos constituyentes no tienen un significado previo a la determinación de su sintaxis lógica (Glock, 1996: 333).

De lo anterior se deriva también la afirmación (Wittgenstein, 1973: $3.332,3.333)$ de que una función proposicional no puede ser su propio argumento, principio que desarma la paradoja de Russell. Pero otra consecuencia del principio es que las proposiciones del propio Tractatus carecen de sentido, ya que "emplean conceptos 
formales ('hecho', 'proposición', 'objeto') para hacer aseveraciones acerca de la esencia de la representación" (Glock, 1996: 334), y como no usan símbolos en una forma significativa, estas proposiciones, de acuerdo con la lógica expuesta en el Tractatus mismo, ni siquiera pueden mostrar lo que tratan de decir. No obstante, dice Glock, el Tractatus hace una diferencia entre sinsentidos que son el resultado de la falta de comprensión de la sintaxis lógica, y, en palabras de Ramsey, "sinsentidos importantes":

El Tractatus no es ni un chiste existencial ni un poema sin sentido extendido, con un sistema de numeración. Su intención era cantarle las golondrinas a la metafísica y sólo viola los límites de sentido para alcanzar el punto de vista lógicamente correcto, que le permita a uno emprender un análisis lógico crítico sin violaciones adicionales (Wittgenstein, 1973: 4.1213, 6.53) (Glock, 1996: 335).

En los párrafos precedentes hemos resumido, con demasiada brevedad y omisiones, la explicación que un estudioso de la filosofía de Wittgenstein como Hans-Johann Glock ofrece para dar cuenta de la distinción ubicua entre decir y mostrar, tal como surge del sistema del Tractatus mismo: estrechamente relacionada con la noción de la bipolaridad esencial de las proposiciones y su posibilidad de servir como retrato de un hecho gracias a su estructura lógica.

Pero según hemos visto, esta explicación no es del todo satisfactoria. No toda esta distinción tiene el mismo estatus ante la lógica; una excepción muy importante es lo inefable místico, que, de acuerdo con el propio Wittgenstein, es la parte más importante del libro, aunque Glock diga de ella burlonamente: "Hay razones para considerar esto como una actuación ligeramente histérica de autopromoción frente a un editor potencial" (1996: 330). 


\section{La distinción entre decir y mostrar y los problemas filosóficos}

Ciertamente, cuando se explica la distinción que nos ocupa en términos de la lógica del Tractatus, entonces, al perder este sistema su vigencia a los ojos de un Wittgenstein más maduro, se pierde también este concepto y su importancia. La distinción entre lo que una proposición, en el sentido del Tractatus, dice y lo que muestra, se extingue junto con el concepto de la proposición tractariana. Pero no es necesario limitar la explicación de esta manera. Más bien, como ya he insinuado, parece un error limitarla así. Trataré de hacer plausible en este apartado por qué pienso esto.

La distinción entre decir y mostrar nace de una preocupación filosófica profunda que, ciertamente, es la que ha inspirado a Wittgenstein a crear el Tractatus, que es anterior a su expresión en el lenguaje lógico de la obra, donde explica la ubicuidad de esta distinción, según Glock reconoce, pero sin poder esencialmente dar cuenta de ella, ya que en su visión parece más una consecuencia colateral del sistema lógico. En su larga lista de "inefabilia", como los llama con ligero tono de burla, Glock enumera desde la forma pictórica que comparten el retrato o lo que es retratado, hasta las proposiciones del propio Tractatus. Pero él nunca parece recordar que el objetivo principal que trata de resolver el Tractatus son los problemas de la filosofía. La intención que ha inspirado la creación del Tractatus es la misma que luego inspira a su autor a derribar este sistema; pero no la distinción entre decir y mostrar, que es una parte importante de ella. De hecho, si parece que esta noción no puede sobrevivir el derribamiento del sistema del Tractatus, entonces es probable que nuestro entendimiento de esta noción haya quedado corto.

Si queremos obtener una comprensión más completa de la fuente filosófica de la que brota esta distinción, no restringida a su visión como parte del sistema lógico presentado en el Tractatus 
- lo que muy probable no sería suficiente-, debemos tener en cuenta lo que Glock no hace con suficiencia: los motivos filosóficos que Wittgenstein tenía en primer lugar para emprender esta obra. El prólogo es bastante elocuente en este sentido: "El libro trata los problemas filosóficos y muestra... que el planteamiento de estos problemas descansa en la incomprensión de la lógica de nuestro lenguaje" (Wittgenstein, 1973: 11). Qué cosa es la filosofía, en términos del Tractatus, lo comenta el autor a partir de la proposición 4.111. En 4.112 nos dice:

El objetivo de la filosofía es la clarificación lógica de los pensamientos. La filosofía no es una doctrina, sino una actividad. Una obra filosófica consta esencialmente de elucidaciones. El resultado de la filosofía no son 'proposiciones filosóficas', sino la aclaración de proposiciones (Wittgenstein, 1973: 4.112).

Igual como en su filosofía posterior, los problemas filosóficos son aquí para Wittgenstein no problemas que pudieran resolverse mediante algún descubrimiento nuevo o mediante la construcción de alguna teoría. Las preguntas filosóficas no son preguntas reales; las preguntas filosóficas no se contestan, sino se disuelven porque nos damos cuenta de que carecen de sentido. Pero esto no quiere decir que son triviales o que carecen de importancia. Así, señala Wittgenstein en Investigaciones filosóficas:

Los problemas que surgen de una malinterpretación de nuestras formas lingüísticas tienen el carácter de lo profundo. Son profundas inquietudes; se enraízan tan profundamente en nosotros como las formas de nuestro lenguaje y su significado es tan grande como la importancia de nuestro lenguaje (Wittgenstein, 1988: 111).

Aunque sea un problema profundo que se manifiesta en las preguntas filosóficas, lo inefable que menciona Glock no tiene el ca- 
rácter de un algo que el lenguaje fuera incapaz de expresar. Contra esta noción, que podría parecer sugestiva, James Conant apunta:

El silencio en que [Wittgenstein] desea dejarnos al final es uno en el cual nada se ha dicho y no hay nada que decir (del que nos habíamos imaginado que hubiera)... El silencio con el cual nos quedamos no es un silencio cargado, que viniera con la postura consciente de guardar lo sagrado de lo inefable (Conant, 1991: 344).

Si aceptamos lo que Conant dice, en la cita anterior, acerca de que no hay inefables, y en vista de lo que el propio Wittgenstein señala sobre la naturaleza de los problemas filosóficos, entonces ¡cómo ha de entenderse lo profundo de estos problemas que no pueden enunciarse propiamente? Esta pregunta, sugiere Marie McGinn, de hecho puede entenderse como la cuestión central de interpretación de toda la filosofía de Wittgenstein (cf. McGinn, 2001: 26). Citando Investigaciones filosóficas 132, nos recuerda que el objetivo de Wittgenstein es establecer un orden en lo que sabemos sobre nuestro uso de lenguaje. Es por ello que no necesitamos ninguna teoría, ni ningún descubrimiento nuevo, sino debemos reflexionar acerca de lo que ya dominamos: nuestro lenguaje. Al poner en orden nuestro conocimiento del lenguaje nos damos cuenta que lo que parecía un problema filosófico no tiene sustento. Con esto en mente, McGinn afirma: "La distinción central en el orden que las elucidaciones de Wittgenstein tratan de llevar a nuestro conocimiento del uso de lenguaje es... la distinción entre decir y mostrar" (2001: 27).

Glock relata, según hemos visto, que Ramsey atribuye a las últimas elucidaciones del Tractatus un "sinsentido importante". Conant piensa que una lectura así implicaría la postulación de algo que un parlante puede intentar decir, pero sin lograrlo, porque las palabras que usa no expresan un pensamiento determinado; noción que Conant rechaza, y también McGinn la encuentra di- 
fícil de aceptar. En lugar de hablar de sinsentidos sustanciosos, McGinn sugiere:

Muy a grandes rasgos, yo deseo conectar la idea de lo que puede ser mostrado pero no puede ser dicho con lo que es esencial para el lenguaje -es decir, con lo que limita o condiciona su sentido- y que por ello es previo a pensamientos que tienen sentido y que son verdaderos o falsos. Lo que sólo puede ser mostrado y no dicho nada tiene que ver con pensamientos (es decir, con verdades) que no pueden ser expresados sin violación de la sintaxis lógica; más bien, se relaciona con las condiciones o límites de sentido que son revelados sólo en nuestro uso real de lenguaje, en las aplicaciones que hacemos de él, es decir, en nuestro uso de las oraciones con sentido. Es esta conexión esencial entre lo que se muestra y que se revela sólo en el uso o la aplicación del lenguaje que hace imposible decir lo que se muestra [...] (2001: 28).

El motivo de la distinción en el Tractatus entonces no se explica en función de características formales de las proposiciones, sino con el orden que Wittgenstein quiere establecer en nuestro conocimiento del uso de lenguaje: conectar la distinción entre lo accidental y meramente posible que se describe en el lenguaje, por un lado; y lo esencial o a priori (cuyo contrario es inconcebible), por el otro, con la distinción entre lo que las oraciones dicen, o lo que muestran.

No debiéramos ver... los comentarios iniciales [del Tractatus] como una serie de afirmaciones metafísicas sobre la naturaleza de una realidad independiente del lenguaje... sino como un retrato material de nuestro lenguaje que Wittgenstein emplea meramente como medio de clarificación (McGinn, 2001: 28).

McGinn elabora este argumento en detalle, recurriendo a varios pasajes de la obra de Wittgenstein para convencernos de que, efectivamente, la distinción entre decir y mostrar depende, ya desde 
el Tractatus, de una cuestión de sentido, independiente de la indispensable bipolaridad de las proposiciones; no podemos seguir su argumento aquí en detalle, pero hace parecer plausible que esta independencia permite conservar la noción en la filosofía posterior de Wittgenstein.

La lógica, sugiere McGinn:

[...] no consiste de un sistema de verdades para que pudiese surgir la cuestión de justificación. Más bien, la lógica, en su totalidad, es coetánea con el fenómeno de lenguaje y el lógico sólo postula una notación en que relaciones de inferencia entre proposiciones, de las cuales cualquiera que entiende el lenguaje ya tiene un dominio práctico, son desplegadas de manera perspicua. En el caso de las leyes lógicas, su aplicación es esencialmente previa a su formulación en forma de una ley. Una vez que tenemos lenguaje en uso, ya tenemos el todo de la lógica... pensar del mundo ya es pensar conforme a las leyes de la lógica (2001: 32).

Ya para el Tractatus, propone McGinn, es verdad que lo que se muestra y no puede ser dicho depende del dominio práctico que tenemos del lenguaje, porque depende de algo que hacemos (usar el lenguaje), y no de algo que sea verdadero o de algo que sepamos. La lógica misma no es un sistema de reglas a priori, sino depende de un dominio previo del lenguaje, según afirma el pasaje recién citado. Es precisamente esta distinción en la que Wittgenstein se apoya en Sobre la certeza, ahora bajo la noción de "observación gramática”,, para rechazar como inútiles los esfuerzos de Moore por refutar el escepticismo.

Su crítica del rechazo de sentido común de Moore contra la duda escéptica tiene que ser vista como un intento de demostrar que Moore - al igual que el filósofo que se propone justificar la lógi$\mathrm{ca}$ - trata una cuestión que se refiere al sentido (uso) de nuestras palabras como si fuera una cuestión que se refiere a hechos. [...] 
Estas así llamadas proposiciones [de Moore] tienen un papel peculiar en el sentido de que son un intento de articular algo que es supuesto en nuestro uso normal del lenguaje, algo que se manifiesta en este uso que es esencial para el sentido de las palabras y que, como parlantes de idioma, ya captamos prácticamente. Este cambio que se presenta en la crítica que Wittgenstein hace de Moore es uno que refleja el cambio en nuestra comprensión del estatus de las proposiciones de la lógica en el Tractatus. El cambio es de una cuestión de verdad — ¿ ¿sabe Moore que esta es una mano? - a una cuestión de sentido - ‘ ¿tiene sentido dudar que esta es una mano?' (McGinn, 2001: 33).

El propósito filosófico de Wittgenstein es hacer evidente que el surgimiento de los problemas filosóficos es parte de la manera en que "habitamos en el lenguaje" y que "no podemos salir de él y darle un fundamento en verdades" (McGinn, 2001: 35). Es así como la distinción entre decir y mostrar es un tema que se comprende bajo el entendido de que de nada sirven teorías e hipótesis para explicar lo que no debe ser explicado, sino pensado como carente de fundamento: los problemas filosóficos.

McGinn sugiere en su discusión con James Conant y Cora Diamond, de la cual provienen los pasajes anteriores, que ellos están en lo correcto al insistir en que debemos leer el Tractatus de una manera que evite atribuirle aseveraciones metafísicas o teoréticas; pero sugiere que este texto hace más que exponer los pronunciamientos filosóficos como sinsentidos. Ella conecta esto último, como hemos visto, con la profundidad que el propio Wittgenstein atribuye a las preocupaciones filosóficas, y con el orden que quiere establecer en nuestra comprensión de lo que sabemos acerca del lenguaje, para lograr, a lo que Conant se refiere, en la parte inicial del pasaje citado unos párrafos arriba: "el objetivo es deshacer nuestra atracción a varias ristras bien formadas de palabras que resuenan con el aura de sentido"; y romper así el 
embrujo de nuestro entendimiento por medio del lenguaje (cf. Wittgenstein, 1988: \$109).

\section{Conclusión}

La explicación de la distinción entre decir y mostrar que ofrece McGinn hace uso de la noción del dominio práctico del lenguaje; y es la imposibilidad de encontrar un fundamento de esta práctica (por ejemplo, en condiciones de verdad) que finalmente es el origen de la distinción wittgensteiniana que aquí nos ocupa. Esta idea concuerda con la afirmación tractariana de que el lenguaje común está en perfecto orden lógico así como está (cf. Wittgenstein, 1973: 5.5563). Lo que todo el sistema del Tractatus a fin de cuentas trata de hacer visible es lo irreal de los problemas filosóficos, y que el origen de éstos es nuestra incapacidad de ver este orden lógico (cf. Wittgenstein, 1973: 4.002).

No estoy insinuando, desde luego, que Glock no esté perfectamente consciente de que ésta sea la meta del Tractatus, sólo que él no parece hacer uso de este motivo en su explicación de la distinción entre decir y mostrar. En su análisis de la noción, él quiere apelar a un principio de explicación (tácito) diferente de la visión wittgensteiniana de la filosofía, uno que prescinde del motivo general de esta filosofía; parece que él quiere que la explicación no dependa de una contextualización así. Esto puede hacer su análisis más apetecible a una moda filosófica que ha archivado a Wittgenstein desde hace tres décadas.

En cuanto a la continuidad de la distinción entre decir y mostrar en la filosofía wittgensteiniana no debemos olvidar, por un lado, que el propio Wittgenstein en su filosofía posterior rechazaba explícitamente la imagen de la escalera que habría que tirar después de escalarla, invocada en las últimas líneas del Tractatus (Glock, 1996: 335). Pero también es cierto que la preocupación 
filosófica que inspiraba estas líneas está vigente en el filosofar wittgensteiniano hasta las últimas líneas que redactaba, como McGinn sugiere en su lectura de Sobre la certeza.

En el fondo, no importa si insistimos en limitar la distinción explícita entre decir y mostrar a la teoría de lenguaje propuesta en el Tractatus o si adoptamos una lectura como la que propone McGinn, mientras estemos conscientes de que la idea filosófica, de la cual esta distinción originalmente brota, es una que permea en toda la filosofía de Wittgenstein; y que, en el primer caso, en nuestro afán por hacer filosofía académica no estemos adoptando una práctica filosófica contraria a la que Wittgenstein nos ha permitido visualizar acerca de las preguntas que tienen, y las que no tienen respuestas, y entonces lo que tiene y lo que no tiene justificación como práctica filosófica.

\section{Bibliografía}

Conant, James, 1991, "Throwing Away the Top of the Ladder", en The Yale Review 79, pp. 328-364; citado según McGinn, 2001.

Dummett, Michael, 1996, Origins of Analytical Philosophy, Harvard University Press, Cambridge, Mass., 200 pp.

Fogelin, Robert J., 1987, Wittgenstein, second edition (edited by Ted Honderich), Routledge, The Arguments of the Philosophers, Londres y Nueva York, 256 pp.

Glock, Hans-Johann, 1996, A Wittgenstein Dictionary, Blackwell, Oxford, 406 pp.

McGinn, Marie, 2001, "Saying and Showing and the Continuity of Wittgenstein's Thought", en The Harvard Review of Philosophy, IX, Cambridge, Mass., pp. 24-36. 
Pitcher, George, 1964, The Philosophy of Wittgenstein, Prentice-Hall, Englewood Cliffs, $340 \mathrm{pp}$.

Rhees, Rush, 1996, Discussions of Wittgenstein, Thoemmes Press, Bristol, $161 \mathrm{pp}$.

Varga von Kibéd, Matthias, 2001, "Variablen im Tractatus", en Ludwig Wittgenstein Tractatus logico-philosophicus, (Herausgegeben von Wilhelm Vossenkuhl), Akademie Verlag, Klassiker Auslegen, Berlin, pp. 209-229.

Wittgenstein, Ludwig, 1973, Tractatus Logico-Philosophicus (trad. de Jacobo Muñoz e Isidoro Reguera), Alianza, Madrid, 215 pp. (la numeración de los pasajes citados se refiere a las secciones).

——, 1988, Investigaciones filosóficas (trad. de Alfonso García Suárez y Ulises Moulines), UNAM, México, 549 pp. (la numeración de los pasajes citados se refiere en la parte I a las secciones, a los números de página en la parte II). 